\title{
Species-Dependent Regulation of Monocyte/Macrophage la Antigen Expression and Antigen Presentation by Prostaglandin E
}

\author{
Steven L. Kunkel, Darrell A. Campbell, Jr., \\ Stephen W. Chensue, AND Gene I. Higashi \\ Departments of Pathology and Surgery, University of Michigan Medical School, and School \\ of Public Health, University of Michigan, Ann Arbor, Michigan 48109
}

Received July 3, 1985; accepted September 12, 1985

\begin{abstract}
The expression of la antigen by various murine and human macrophage populations and the ability of prostaglandins of the $E$ series to regulate la antigen expression were explored. Monocytes and macrophages from human and murine populations demonstrated a dichotomy in the expression of Ia antigen. Both human monocytes and macrophages expressed elevated levels of Ia antigen compared to their murine counterpart. Murine macrophages appear to express elevated levels of Ia antigen only when actively interacting with $\mathrm{T}$ lymphocytes in vivo or with lymphokines in vitro. Prostaglandins of the $\mathrm{E}$ series can suppress murine macrophage la antigen expression, but have little effect on the expression of Ia antigen by human monocytes and macrophages. Also, prostaglandins of the $E$ series do not modulate the ability of human monocytes to present antigen to autologous lymphocytes when studied over a broad concentration range. These data suggest that prostaglandin $\mathrm{F}$ compounds do not profoundly affect human monocyte/macrophage Ia antigen expression or human monocyte antigen presenting activity. (9) 1986 Academic Press, Inc.
\end{abstract}

\section{INTRODUCTION}

Metabolites of arachidonic acid are known to serve as potent regulatory compounds during episodes of acute and chronic inflammation (1-3). These lipid metabolites synthesized by cells at foci of inflammation, via both the lipoxygenase and cyclooxygenase pathways, may exert their modulating activity by dictating immune cell activity (3-5). This observation has been recently supported by experiments demonstrating the ability of prostaglandins of the E series to influence macrophage/lymphocyte interaction, the key components in cell-mediated immune mechanisms $(3,6)$. In studies from our laboratory we demonstrated that $\mathrm{PGE}_{1}$ and $\mathrm{PGF}_{2 \alpha}$ significantly influenced the in vivo kinetics of I-region-associated (Ia) antigen expression on murine immune granuloma macrophages (6), while investigations by Steeg et al. (7) showed that PGE can suppress lymphokine-induced la antigen expression by murine peritoneal macrophages. One of the major concerns of investigations involving mouse macrophages is the applicability of the data to human macrophage/monocyte systems. In the present study, we have investigated the effect of PGE compounds on Ia antigen expression and antigen presenting activity by human monocytes and have correlated these findings with the modulation of murine macrophage Ia antigen expression. 


\section{MATERIALS AND METHODS}

Isolation of blood mononuclear cells. Human and murine (CBA/J-H-2 $\left.{ }^{\mathrm{k}}\right)$ mononuclear cells were isolated from anticoagulant blood using Ficoll-Hypaque density gradients, as previously described (8). Additional human venous blood was drawn for the isolation of autologous serum. The isolation of murine granuloma macrophages and preparation of murine lymphokine(s) were carried out as previously described (6). In addition, human alveolar macrophages were recovered and cultured using the procedure of Razma et al. (9).

Culture of human and mouse mononuclear phagocytic cell populations. Mononuclear cells from the gradient interface were thoroughly washed with RPMI-1640 (Grand Island Biochemical, Inc., Grand Island, N.Y.) and finally resuspended in complete medium consisting of RPMI-1640 with $5 \%$ autologous serum and $10 \mu \mathrm{g} / \mathrm{ml}$ gentamicin. The cells were applied to either $35-\mathrm{mm}$ plastic culture plates (Corning Glass Works, Corning, N.Y.) or glass tissue culture chambers (Lab Tek, Miles Laboratories, Inc., Naperville, Ill.), the latter for the determination of Ia antigen expression. After adherence for $4 \mathrm{hr}$ at $37^{\circ} \mathrm{C}$, the nonadherent cells were removed by washing with warm RPMI-1640 and the adherent cells were either examined for Ia antigen expression or returned to the incubator in complete medium in the presence or absence of $1 \mu M$ $\mathrm{PGE}_{1}$ (gift from Dr. John Pike, The Upjohn Co., Kalamazoo, Mich.).

Assay for Ia antigen expression. All monocyte and macrophage populations were freshly isolated and allowed to adhere for $4 \mathrm{hr}$ at $37^{\circ} \mathrm{C}$ in a $5 \% \mathrm{CO}_{2}$ humidified atmosphere prior to assaying for initial Ia antigen expression. Adherent mononuclear phagocytic cells were quantitated and identified as macrophages by morphologic criteria, ability to ingest latex beads, and nonspecific esterase staining. After fixation with $0.1 \%$ paraformaldehyde, all cells were washed with phosphate-buffered saline (PBS) containing $5 \%$ fetal calf serum (FCS) and $0.05 \%$ sodium azide. The human cells were then reacted with Ia monoclonal antibody (9) for $30 \mathrm{~min}$, washed with PBS-5\% FCS$0.05 \%$ sodium azide, and developed with fluoroscein labeled $\mathrm{F}\left(\mathrm{ab}^{\prime}\right)_{2}$ rabbit IgG directed against mouse IgG. Controls included an anti-B-cell antibody unreactive with macrophages (10) and a murine monoclonal antibody directed against $\mathrm{Ia}^{\mathrm{b}}$ (American Type Culture Collection, Bethesda, Md.). Ia antigen expression by murine macrophages was assessed using monoclonal antibody directed against $\mathrm{I}-\mathrm{A}^{\mathrm{k}}$ described by $\mathrm{O} i$ et al. (11). Monoclonal anti I-A ${ }^{\mathrm{b}}$, described by Ozato and Sacks (12), was used as a control. Clones synthesizing both murine I-A antibodies were purchased from the American Type Culture Collection. Fluoroscein-conjugated $\mathrm{F}\left(\mathrm{ab}^{\prime}\right)_{2}$ rabbit IgG directed against mouse IgG was used to label the bound I-A antibody. By ultraviolet microscopy a minimum of 200 cells were counted to determine the percentage of positive cells.

Assay for antigen presentation. Adherent human blood monocytes were isolated as above, while nylon wool-purified $T$ cells were prepared using a method previously described (13). Adherent cells $\left(6 \times 10^{4}\right)$ were incubated in the wells of a microtest plate in the presence of none, or various concentrations of $\mathrm{PGE}_{1}$, and $25 \mu \mathrm{g}$ tetanus toxoid. Cells were incubated in a $37^{\circ} \mathrm{C} 5 \mathrm{CO}_{2}$ humidified incubator. After 4 days incubation, the cells were washed and $2 \times 10^{5}$ nylon wool-purified T cells were added. At the end of a further 5-day incubation period, each well was pulsed with $2 \mu \mathrm{Ci}$ of $\left[{ }^{3} \mathrm{H}\right]$ thymidine (sp act $6.7 \mathrm{Ci} / \mathrm{mmole}$; New England Nuclear, Boston, Mass.). After 18 $\mathrm{hr}$, cultures were harvested using a multiple automated sample harvester (MASH I). Scintillation counting was performed in a Beckman liquid scintillation counter. 


\section{RESULTS}

Ia antigen expression by various human and mouse macrophage populations. The macrophage is known to be a key effector cell in the generation and maintenance of various chronic immune responses (14). The initiation of these macrophage/lymphocyte-mediated cellular responses is dependent upon many molecular signals that can interact in an amplification loop leading to immune cell activation. Signals of lymphocyte origin include various protein and peptide cytokines, while those of macrophage origin include arachidonate metabolites, interleukin 1, and antigen class II (Ia) molecule presentation structures $(3,4,15)$. Therefore, we initially wished to profile the capacity of various human and mouse macrophage populations to express Ia antigen. As shown in Table 1, a dichotomy exists between human and mouse macrophage Ia antigen expression. Normal mouse monocyte and macrophage populations expressed very low levels of Ia antigen unless specifically interacting with $\mathrm{T}$ lymphocytes, such as the case in hypersensitivity granulomatous inflammation. Conversely, human monocyte/macrophages from various sources normally express high levels of Ia-like antigen that do not significantly change during an inflammatory response (Table 1).

Modulation of la-like antigen on mouse but not human macrophages by $P G E_{l}$. Previous studies from our laboratory have demonstrated that in vitro $\mathrm{PGE}_{1}$ treatment of murine macrophages can directly affect Ia antigen expression (6). Although the reduction in Ia antigen expression by lymphokine-treated murine macrophages at Days 1 and 2 followed by a rebound at Days 3 and 4 corroborates the findings of Beller and $\mathrm{Ho}(16)$, we have now shown that $\mathrm{PGE}_{1}$ can modulate this expression of lymphokine-induced Ia antigen. As shown in Table $2, \mathrm{PGE}_{1}(1 \mu M)$ can suppress mouse Ia antigen expression 10-fold, while the percentage of antigen expression by human cells remained unaltered during the 4-day study period. In additional experiments, the expression of Ia antigen by mouse macrophages treated with lymphokine at time 0 and $\mathrm{PGE}_{1}(1 \mu M)$ at Day 3 resulted in a reduction of macrophage la antigen expression on Day 4. Withholding $\mathrm{PGE}_{1}$ treatment from the human macrophages until Day 3 had no effect on the expression of Ia-like antigen on Day 4. The refractory response of antigen expression by human macrophages to $\mathrm{PGE}_{1}$ was also demonstrated using human alveolar and human peritoneal macrophages (data not shown). In addition, $\mathrm{PGE}_{2}$ had the identical effect as $\mathrm{PGE}_{1}$ in the above system.

\section{TABLE 1}

Percentage of Macrophages Expressing Ia Antigen

\begin{tabular}{lcc}
\hline \multicolumn{1}{c}{ M $\phi$ source } & Human & Mouse \\
\hline Blood & $65 \pm 15$ & $7 \pm 4$ \\
Peritoneum & $69 \pm 21$ & $<5$ \\
Alveolar & $59 \pm 19$ & $<5$ \\
Pulmonary hypersensitivity granuloma & $80 \pm 16^{a}$ & $56 \pm 7^{b}$ \\
\hline
\end{tabular}

Note. Comparison of the percentage of Ia antigen expression between freshly isolated human and mouse macrophages prepared from various sources. Means \pm SEM of four to seven experiments. Each experiment was performed in triplicate.

${ }^{a}$ Granuloma macrophages were isolated from patients with pulmonary granulomatous sarcoidosis.

${ }^{b}$ Granuloma macrophages were isolated from experimental murine schistosome egg delayed-type hypersensitivity pulmonary granulomatous lesions. 
TABLE 2

Percentage of Macrophages Expressing Ia-like Antigen

\begin{tabular}{|c|c|c|c|c|}
\hline \multirow{2}{*}{$\begin{array}{l}\text { Time } \\
\text { (hr) }\end{array}$} & \multicolumn{2}{|c|}{ Human } & \multicolumn{2}{|c|}{ Mouse $^{a}$} \\
\hline & $-\mathrm{PGE}_{1}$ & $+\mathrm{PGE}_{1}\left(10^{-6} M\right)$ & $-\mathrm{PGE}_{1}$ & $+\mathrm{PGE}_{1}\left(10^{-6} M\right)$ \\
\hline 4 & $59 \pm 3$ & - & $51 \pm 4$ & - \\
\hline 24 & $64 \pm 5$ & $71 \pm 8$ & $21 \pm 3$ & $18 \pm 6$ \\
\hline 48 & $61 \pm 9$ & $75 \pm 10$ & $13 \pm 5$ & $10 \pm 4$ \\
\hline 72 & $78 \pm 11$ & $71 \pm 5$ & $35 \pm 11$ & $<5$ \\
\hline 96 & $61 \pm 13$ & $65 \pm 8$ & $49 \pm 9$ & $<5$ \\
\hline
\end{tabular}

Note. Effect of $\mathrm{PGE}_{1}$ on the expression of Ia-like antigen by human peripheral blood monocytes and murine macrophages. $\mathrm{PGE}_{1}$ did not significantly alter the expression of Ia-like antigen by human cells during the 4-day study period, while Ia expression on granuloma macrophages from the mouse was dramatically reduced by $\mathrm{PGE}_{1}$. Means \pm SEM of three experiments. Each experiment was performed in triplicate.

${ }^{a}$ Mouse macrophages were incubated in the presence of mouse spleen cell derived lymphokines from time 0 in order for Ia antigen to be reexpressed at Days 3 and 4; this expression was blocked by PGE .

Inability of $P G E_{1}$ to modulate antigen presenting function by human monocytes. The above studies demonstrated that $\mathrm{PGE}_{1}$ does not appear to influence the expression of Ia antigen by cultured human monocytes. In order to assess the ability of $\mathrm{PGE}_{1}$ to alter the functional role of Ia antigen-positive human monocytes, we examined the ability of this biologically active lipid to modulate monocyte-induced lymphocyte proliferation. Human monocytes were incubated in the presence of tetanus toxoid and various concentrations of $\mathrm{PGE}_{1}$ for $96 \mathrm{hr}$. At this point the adherent cells were washed, and nylon wool-purified $T$ cells wee added to culture such that the final ratio of adherent cells to $T$ cells was 1:3. As shown in Fig. 1, $\mathrm{PGE}_{1}$ at doses ranging from $1 \times 10^{-6}$ to $1 \times 10^{-9} \mathrm{M}$ did not inhibit the ability of human monocytes to present antigen to $T$ lymphocytes. The strict requirement for monocytes to express Ia antigen in order for lymphocytes to proliferate was demonstrated by the suppression of proliferation when monocytes were pretreated with antibody directed against the Ia antigen. Monocytes pretreated with antibody directed against Ia antigen and then used as antigen presenting cells could only induce $10-20 \%$ of the normal lymphocyte proliferation as untreated monocytes. These studies suggest that PGE may not play a modulatory role in human monocyte/macrophage Ia antigen expression or antigen presentation.

\section{DISCUSSION}

The mobilization of arachidonic acid from membrane-bound phospholipids and the conversion of this substrate via the lipoxygenase and/or cyclooxygenase pathways yield products with diverse biological activities $(2,17)$. Recent studies have demonstrated that products from the cyclooxygenase pathway can influence the development and regulation of immune responses and inflammatory reactions (17). These investigations have centered around the in vivo use of prostaglandins of the E series to suppress acute and chronic inflammatory responses in experimental animals. Although the exact means by which PGE compounds attenuate inflammatory reactions are not fully understood, it is known the PGEs can down-regulate murine macrophage and 


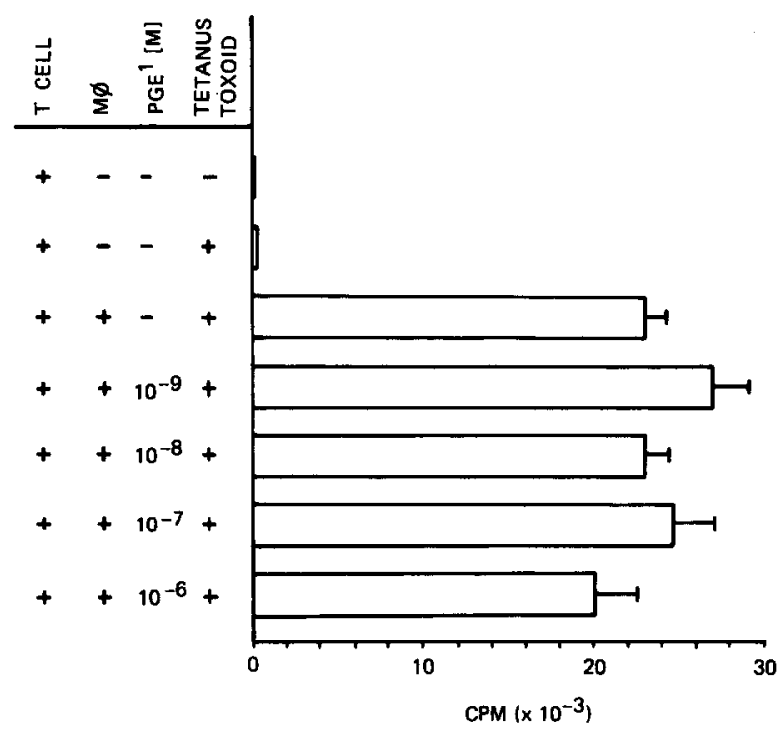

FIG. 1. Effect of various doses of $\mathrm{PGE}_{1}$ on antigen presentation by adherent monocytes. Adherent cells

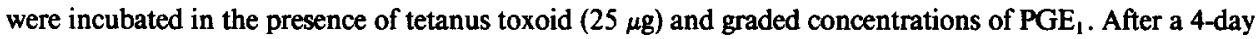
incubation period the cells were washed and then cocultivated with $T$ cells for 6 additional days. To demonstrate that the T-cell preparation was adequately depleted of monocytes, column-purified $T$ cells were incubated for 6 days in the presence of tetanus toxoid but no additional source of monocytes (line 2 ).

lymphocyte function (18). However, few studies have closely examined the ability of prostaglandins of the $E$ series to regulate human monocyte/macrophage function(s) necessary to interact with $\mathrm{T}$ lymphocytes. In the present report we have examined the expression of Ia antigen by various mouse and human macrophage populations and have studied the ability of PGEs to regulate the expression of Ia antigen. In addition, the antigen presenting activity of cultured human monocytes was assessed in the presence and absence of PGEs.

Our results have demonstrated a dichotomy in the expression of Ia antigen between various human and murine macrophage populations. It is noteworthy that both human monocyte and macrophage populations express high levels of Ia antigen compared to their murine counterparts. Murine macrophages apparently only express high levels of Ia antigen in vivo when actively interacting with $\mathrm{T}$ lymphocytes, as would be the case during delayed-type hypersensitivity reactions. Contrary to human macrophages, Ia antigen expression by murine macrophages in vitro is rapidly lost but can be reexpressed by incubating with lymphokine preparations.

In an extension of our earlier observations we provide evidence that PGEs can suppress murine macrophage la antigen expression in the preence of lymphokine, but PGEs do not alter the expression of Ia antigen by human monocytes and macrophages. In addition, PGEs do not modulate the ability of human monocytes to present antigen to autologous $\mathrm{T}$ lymphocytes when examined over a $4 \log$ concentration range. Our results confirm and extend the studies of Mori and Hayward (19), whereby cultured human monocytes retained their ability to present antigen in association with Ia surface antigen. The persistence of Ia antigen expression by human monocytes may in part be due to their reduced rate of surface Ia antigen turnover (20). The mechanism(s) by 
which human, but not murine, monocyte/macrophages remain refractory to the effects of PGEs with regard to Ia antigen expression and antigen presenting activity is not clear, but may be due to the in vivo interaction of macrophages with T-lymphocyte product(s) resulting in an altered state of immunological competence. Recent studies have demonstrated that human monocytes contaminated with $\mathrm{T}$ lymphocytes can express high levels of Ia-like antigen for up to 5 days (21), but thorough depletion of the $\mathrm{T}$ lymphocytes resulted in a decrease in the expression of Ia-like antigen. This latter phenomenon was reversed when $\gamma$-interferon was substituted for the T lymphocytes. In addition, PGEs have also been shown to down-regulate murine macrophage function, as related to tumoricidal activity, but this suppression was not observed if macrophages were incubated with murine $\gamma$-interferon, a potent lymphokine, prior to PGE exposure (22). Therefore, we feel that the continued expression of Ia-like antigen and the potential for antigen presentation by human monocytes and macrophages, even in the presence of PGEs, may reflect prior stimulation with T-lymphocyte product(s) that is tonically secreted by human $T$ cells. Further analyses are currently underway to delineate the mechanisms whereby other arachidonic acid metabolites may dictate macrophage/lymphocyte interactions.

\section{ACKNOWLEDGMENTS}

The authors express their appreciation for the expert secretarial support of Jeny Brown. This work was supported in part by NIH Grants HL-31237 and HL-31963. Part of this work was performed during the tenure of an established investigator award (S.L.K.) from the American Heart Association.

\section{REFERENCES}

1. Ford-Hutchinson, A. W., Bray, M. A., Doig, M. U., Shipley, M. E., and Smith, M. J. N., Nature (London) 286, 264, 1980.

2. Kunkel, S. L., and Chensue, S. W., Adv. Inflammation Res. 7, 93, 1984.

3. Kunkel, S. L., Chensue, S. W., and Higashi, G. I., J. Clin. Invest. 74, 514, 1984.

4. Snyder, D. S., Beller, D. I., and Unanue, E. R., Nature (London) 299, 163, 1982.

5. Klickstein, L. B., Shapleigh, C., and Goetzl, E., J. Clin. Invest. 66, 1166, 1980.

6. Kunkel, S. L., Chensue, S. W., Plewa, M., and Higashi, G. I., Amer. J. Pathol. 114, $240,1984$.

7. Steeg, P. S., Johnson, H. M., and Oppenheim, J. J., J. Immunol. 129, 2402, 1982.

8. Boyum, A., Scand. J. Clin. Lab. Invest. 21, 77, 1968.

9. Razma, A. G., Lynch, J. P., Wilson, B. S., Ward, P. A., and Kunkel, S. L., Amer. Rev. Resp. Dis. 129, $419,1984$.

10. Wilson, B. S., and Kay, N. E., Fed. Proc. 42, 433, 1983.

11. Oi, V. T., Jones, P. P., Goding, J. W., Herzenberg, L. A., and Herzenberg, L. A., Curr. Top. Microbiol. Immunol. 81, 115, 1978.

12. Ozato, K., and Sacks, D. H., J. Immunol. 126, 317, 1981.

13. Julius, M. H., Simpson, I. E., and Herzenberg, L. A., Eur. J. Immunol. 3, 645, 1973.

14. Adams, D. O., and Hamilton, T. A., Annu. Rev. Immunol. 2, 283, 1984.

15. Scala, G., and Oppenheim, J. J., J. Immunol. 131, $1160,1983$.

16. Beller, D. I., and Ho, K., J. Immunol. 129, 971, 1982.

17. Goodwin, J. S., and Webb, D. R., Clin. Immunol. Immunopathol. 15, 106, 1980.

18. Leung, K. H., Ehrke, M. J., and Mihich, E., Int. J. Immunopharmacol. 4, 195, 1982.

19. Mori, M., and Hayward, A. P., Clin. Immunol. Immunopathol. 30, 387, 1984.

20. Smith, B. R., and Ault, K. A., J. Immunol. 127, 2020, 1981.

21. Sztein, M. B., Steeg, P. S., Johnson, H. M., and Oppenheim, J. J., J. Clin. Invest. 73, 556, 1984.

22. Russel, S. W., and Pace, J. L., Mol. Immunol. 21, $249,1984$. 